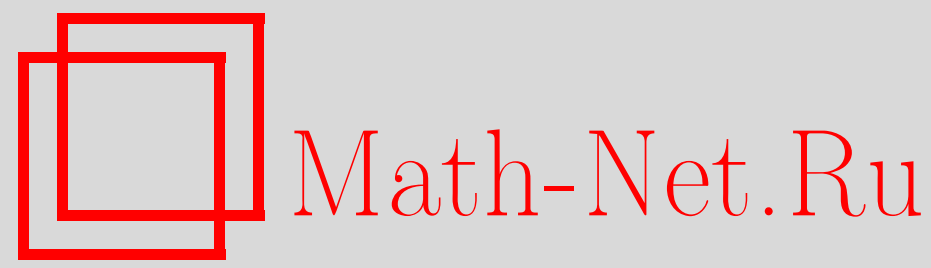

В. А. Горькавый, Восстановление подмногообразия евклидова пространства по вырожденному в линию грассманову образу, Матем. заметки, 1996, том 59, выпуск 5, 681-691

DOI: https://doi.org/10.4213/mzm1762

Использование Общероссийского математического портала MathNet.Ru подразумевает, что вы прочитали и согласны с пользовательским соглашением

http://www. mathnet.ru/rus/agreement

Параметры загрузки:

IP : 35.174 .16 .151

26 апреля 2023 г., 18:11:29

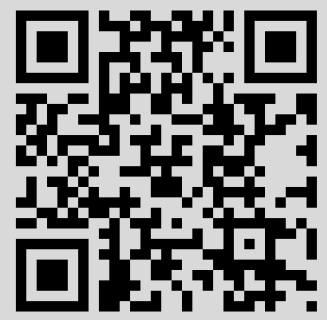




\section{ЕВКЛИДОВА ПРОСТРАНСТВА ПО ВЫРОЖДЕННОМУ \\ В ЛИНИЮ ГРАССМАНОВУ ОБРАЗУ}

\section{В. А. Горькавый}

1. Пусть $F^{n} \subset E^{n+r}$ - ориентируемое регулярное $n$-мерное подмногообразие $(n+r)$-мерного евклидова пространства. Каждой точке $Q \in F^{n}$ поставим в соответствие ориентированное подпространство $E^{r} \subset E^{n+r}$, параллельное ориентированному нормальному пространству $N_{Q} F^{n}$ подмногообразия $F^{n}$ в точке $Q$ и одинаково ориентированное с ним. Такое соответствие задает отображение $G$ из $F^{n}$ в грассманово многообразие $G^{+}(r, n+r)$ ориентированных $r$-мерных подпространств $(n+r)$-мерного евклидова пространства. Образ $G\left(F^{n}\right)$ называют грассмановым образом подмногообразия $F^{n} \subset E^{n+r}$. Будем обозначать его $Г$. В общем случае размерность $Г$ равна размерности $F^{n}$.

Ю.А. Аминовым в [1] была сформулирована следующая общая задача: по заданному регулярному подмногообразию $\Gamma \subset G^{+}(r, n+r)$ найти регулярное подмногообразие $F^{n}$ в $E^{n+r}$, имеющее $\Gamma^{n}$ своим грассмановым образом. Наиболее плодотворно данная проблема изучалась Ю. А. Аминовым [1], [2], Хоффманом и Оссерманом [3] в случае двумерных подмногообразий.

В некоторых случаях грассманов образ Г может иметь размерность меньшую, чем размерность поверхности $F^{n}$ : Это происходит в тех случаях, когда нормальное пространство $F^{n}$ в $E^{n+r}$ вдоль некоторых подмногообразий на $F^{n}$ стационарно. Грассманов образ называют вырожденным, а само подмногообразие $F^{n}$ - тангенциально вырожденным. Тангенциально вырожденные поверхности являются сильно параболическими (в смысле [4]). Проблема восстановления подмногообразия $F^{n} \subset E^{n+r}$ по вырожденному грассманову образу рассматривалась Ю. А. Аминовым и Т. С. Тарасовой. Ими доказана

Теорема [5]. Для того, чтобы кривая $Г \subset G^{+}(2,4)$ была вырожденным грассмановым образом некоторой поверхности $F^{2} \subset E^{4}$, не- 
обходимо и достаточно, чтобы Г была асимптотической линией гиперповерхности $G^{+}(2,4)$, вложенной в сферу $S^{5}$ с помощью плюккеровых координат.

В настоящей работе доказывается общее утверждение.

ТЕоРемА. 1. Если регулярная кривая Г в грассмановом многообразии $G^{+}(r, n+r)$ является грассмановым образом регулярного $C^{p}$, $p>2$, подмногообразия $F^{n} \subset E^{n+r}$, то тогда $\Gamma$ - асимптотическая кривая многообразия $G^{+}(r, n+r) \subset S^{N-1}$.

2. Пусть $\Gamma-$ регулярная $C^{p}, p>1$, асимптотическая кривая в $G^{+}(r, n+r) \subset S^{N-1}$. Тогда для любой точки $P \in \Gamma$ существует окрестность $U \subset \Gamma$ и регулярное $C^{p+1}$ подмногообразие $F^{n} \subset E^{n+r}$, для которого $U-$ грассманов образ.

Здесь и далее рассматривается вложение $G^{+}(r, n+r)$ в сферу $S^{N-1}$ радиуса $1, N=C_{n+r}^{r}$, с помощью плюккеровьг координат.

Напомним, что линия $\Gamma \subset G^{+}(r, n+r)$ - асимптотическая для $G^{+}(r, n+r)$, если в каждой точке $Q \in \Gamma$ в направлении, касательном к $\Gamma$, вторая квадратичная форма $G^{+}(r, n+r) \subset S^{N-1}$ относительно любой нормали равна нулю.

Как следствие доказано известное утверждение о строении поверхности с вырожденным грассмановым образом.

СЛЕДСТВИЕ. Пусть регулярное $C^{p}, \quad p>2$, подмногообразие $F^{n} \subset E^{n+r}$ имеет вырожденный в линию грассманов образ. Тогда радиус-вектор $F^{n} \subset E^{n+r}$ в некоторой локальной системе координат имеет вид

$$
\overrightarrow{\mathbf{r}}\left(v^{1}, \ldots, v^{n}\right)=\vec{\omega}_{1}+\vec{\omega}_{2} \cdot v^{2}+\cdots+\vec{\omega}_{n} \cdot v^{n},
$$

где $\vec{\omega}_{i}$ - вектор-функции, зависящие только от $v^{1}$, и нормальная плоскость $N_{Q} F^{n}$ вдоль подмногообразия $v^{1}=$ const стационарна.

Отметим, что поверхности с вырожденным в линию грассмановым образом являются развертывающимися, т.е. изометричньги пространству $E^{n}$. Отметим также, что содержание теоремы можно сформулировать без применения понятия ориентации заменив $G^{+}(r, n+r)$ грассмановым многообразием $G(r, n+r)$ неориентированных $r$-мерных подпространств в $E^{n+r}$; соответственно $G(r, n+r)$ вкладьвается с помошью плюккеровых координат в проективное пространство.

2. Зафиксируем в $E^{n+r}$ базис $e_{1}, \ldots, e_{n+r}$ и рассмотрим плоскость $E_{0}^{r}=\operatorname{span}\left(e_{1}, \ldots, r_{r}\right)$, которую считаем положительно ориентированной. Подпространства $E^{r}$, проектируюшиеся без вырождения с сохранением ориентации на $E_{0}^{r}$, образуют окрестность точки $P$, соответствующей $E_{0}^{r}$, 
в $G^{+}(r, n+r)$. Каждое такое подпространство $E^{r}$ можно задать в декартовых координатах $x^{i}$ в $E^{n+r}$ в виде

$$
\left(\begin{array}{c}
x^{r+1} \\
\vdots \\
x^{r+n}
\end{array}\right)=\left(\begin{array}{ccc}
z_{1}^{1} & \ldots & z_{r}^{1} \\
\vdots & \ddots & \vdots \\
z_{1}^{n} & \ldots & z_{r}^{n}
\end{array}\right) \cdot\left(\begin{array}{c}
x^{1} \\
\vdots \\
x^{r}
\end{array}\right)
$$

и каждоеподпространство $E^{r}$ натянуто на векторы $\varepsilon_{j}=e_{j}+\sum_{i=1}^{n} z_{j}^{i} e_{r+i}$. Набор чисел $z_{j}^{i}, i=\overline{1, n}, j=\overline{1, r}$, является локальными координатами на $G^{+}(r, n+r)$ и, таким образом, на $G^{+}(r, n+r)$ вводится структура аналитического многообразия [6, с. 174]. Будем обозначать точку из $G^{+}(r, n+r)$ и вектор из касательного пространства $T_{Q} G^{+}(r, n+r)$ матрицей $n \times r$. Для $G^{+}(r, n+r)$ существует вложение в сферу $S^{N-1}$ радиуса 1 с помощью плюккеровых координат $\left(N=C_{n+r}^{r}-\right.$ биномиальньй коэффициент), а именно: каждому ориентированному подпространству, натянутому на векторы $\varepsilon_{i}, i=\overline{1, r}$, ставится в соответствие точка в $E^{N}$ с радиус-вектором $R=R_{1} /\left|R_{1}\right|$, где $R_{1}=\left[\varepsilon_{1}, \ldots, \varepsilon_{r}\right]$ - ориентированньй поливектор. Плюккерово вложение изометрично и эквивалентно [7].

3. Рассмотрим в $G^{+}(r, n+r)$ множество $M r$-мерных подпространств, содержащих $(r-1)$-мерное фиксированное подпространство $E^{r-1}$. Предположим, что $E^{r-1}$ натянуто на векторы $b_{\alpha}=\left(b_{\alpha}^{1}, \ldots, b_{\alpha}^{n+r}\right)$. Тогда $M-$ подмногообразие в $G^{+}(r, n+r)$, задаваемое в локальных координатах

$$
\left(\begin{array}{c}
b_{\alpha}^{r+1} \\
\vdots \\
b_{\alpha}^{r+n}
\end{array}\right)=\left(z_{j}^{i}\right) \cdot\left(\begin{array}{c}
b_{\alpha}^{1} \\
\vdots \\
b_{\alpha}^{r}
\end{array}\right), \quad \alpha=\overline{1, r-1} .
$$

Через каждую точку на $G^{+}(r, n+r)$ проходит $(r-1)$-параметрическое семейство подмногообразий $M$ вида (3.1). В [8] указано, что подмногообразие $M \subset G^{+}(r, n+r)$ является вполне геодезическим и изометричным $n$-мерной сфере радиуса 1 . Как и в [8] будем называть $M$ стандартной сферой $S^{n}$ в $G^{+}(r, n+r)$.

Касательньй вектор $T$ стандартной сферы (3.1) в точке $Q$ удовлетворяет в $T_{Q} G^{+}(r, n+r)$ условию

$$
T\left(\begin{array}{c}
b_{\alpha}^{1} \\
\vdots \\
b_{\alpha}^{r}
\end{array}\right)=0, \quad \alpha=\overline{1, r-1}
$$

Если обозначить через $T_{i}$ вектор-столбцы матрицы $T$, то $(3.2)$ в общем случае есть $r-1$ независимое линейное соотношение между $r$ векторами $T_{i}$, и поэтому (3.2) эквивалентно колинеарности вектор-столбцов $T_{i}$, т.е.

$$
\left[T_{i}, T_{j}\right]=0, \quad i, j=\overline{1, r},
$$


где $\left[T_{i}, T_{j}\right]$ - простой бивектор в евклидовом пространстве. Обратно, легко видеть, что если в $T_{Q} G^{+}(r, n+r)$ задать направление $T$, удовлетворяющее (3.3), то однозначно восстанавливается стандартная сфера $S^{n}$, проходящая через точку $Q$ и такая, что $T \in T_{Q} S^{n}$. Таким образом, (3.3) является характеризационным свойством направлений в $T_{Q} G^{+}(r, n+r)$, касательных к стандартным сферам $S^{n}$. В заключение отметим, что из (3.2) легко получить, что касательные пространства к различньм стандартным с ферам $S^{n}$ не имеют общих направлений и образуют в $T_{Q} G^{+}(r, n+r)$ $(r+n-1)$-мерньй конус $K_{Q}$, задаваемьй в $T_{Q} G^{+}(r, n+r)$ системой условий (3.3).

Лемма 1. Направления, касательные к стандартным сферам, проходящим через точку $P$, и только они в $T_{P} G^{+}(r, n+r)$ являются асимптотическими для $G^{+}(r, n+r) \subset S^{N-1}$.

ДокАЗАТЕЛЬСтво. Пусть $z=\left\|z_{j}^{i}\right\|$ - матрища $n \times r$ локальных координат точки $Q \in G^{+}(r, n+r)$. Соответствующее подпространство $E^{r} \subset E^{n+r}$ натянуто на векторы (см. п. 2):

$$
\varepsilon_{\alpha}=\left(\delta_{\alpha}^{1}, \ldots, \delta_{\alpha}^{r}, z_{\alpha}^{1}, \ldots, z_{\alpha}^{n}\right), \quad \alpha=\overline{1, r}
$$

Радиус-вектор $G^{+}(r, n+r)$, вложенного в $E^{N}$ с помошью плюккеровых координат, имеет вид $R=R_{1} /\left|R_{1}\right|$ (см. п. 2), где $N$-мерный вектор $R_{1}$ в силу вида векторов $\varepsilon_{\alpha}$ имеет следующую структуру: $R_{1}=\left(1 ; \Omega_{1} ; \Omega_{2} ; \Omega_{3}\right)$, где $\Omega_{1}$ - набор координат $(-1)^{r+j} z_{j}^{i}, i=\overline{1, n}, j=\overline{1, r}, \Omega_{2}$ - набор координат $(-1)^{\sigma}\left(z_{t}^{i} z_{h}^{j}-z_{h}^{i} z_{t}^{j}\right), i, j=\overline{1, n}, t, h=\overline{1, r}, \Omega_{3}$ - набор координат, являющихся однородными многочленами степени больше 2 от $z_{j}^{i}$. Имеем

$$
\begin{gathered}
\frac{\partial R}{\partial z_{t}^{i}}=\frac{\partial R_{1}}{\partial z_{t}^{i}} \frac{1}{\left|R_{1}\right|}-\left(R_{1}, \frac{\partial R_{1}}{\partial z_{t}^{i}}\right) \frac{R_{1}}{\left|R_{1}\right|^{3}} \\
\frac{\partial^{2} R}{\partial z_{t}^{i} \partial z_{h}^{j}}=\frac{\partial^{2} R_{1}}{\partial z_{t}^{i} \partial z_{h}^{j}} \frac{1}{\left|R_{1}\right|}-\frac{1}{\left|R_{1}\right|^{3}}\left\{\left(R_{1}, \frac{\partial R_{1}}{\partial z_{h}^{j}}\right) \frac{\partial R_{1}}{\partial z_{t}^{i}}+\left(R_{1}, \frac{\partial R_{1}}{\partial z_{t}^{i}}\right) \frac{\partial R_{1}}{\partial z_{h}^{j}}\right\} \\
-\left\{\left(\frac{\partial R_{1}}{\partial z_{t}^{i}}, \frac{\partial R_{1}}{\partial z_{h}^{j}}\right)-\left(R_{1}, \frac{\partial^{2} R_{1}}{\partial z_{t}^{i} \partial z_{h}^{j}}\right)\right\} \frac{R_{1}}{\left|R_{1}\right|^{3}} \\
+3\left(R_{1}, \frac{\partial R_{1}}{\partial z_{t}^{i}}\right)\left(R_{1}, \frac{\partial R_{1}}{\partial z_{h}^{j}}\right) \frac{R_{1}}{\left|R_{1}\right|^{5}} .
\end{gathered}
$$

Возьмем произвольную точку $P$ в $G^{+}(r, n+r)$. Выберем локальные координаты $z_{j}^{i}$ так, чтобы точке $P$ отвечали значения $z_{j}^{i}=0$. (Для этого в исходном пространстве $E^{n+r}$ необходимо выбрать ортонормированньй 
базис $e_{1}, \ldots, e_{n+r}$ так, чтобы соответствующее точке $P$ подпространство $E^{r} \subset E^{n+r}$ было натянуто на $\left.e_{1}, \ldots, e_{r}.\right)$ Тогда в точке $P$ :

$$
\begin{gathered}
R_{1}=(1 ; 0, \ldots, 0 ; 0, \ldots, 0 ; 0, \ldots, 0), \\
\frac{\partial R_{1}}{\partial z_{t}^{i}}=\left(0 ; 0, \ldots,(-1)^{r+t}, \ldots, 0 ; 0, \ldots, 0 ; 0, \ldots, 0\right), \\
\frac{\partial^{2} R_{1}}{\partial z_{t}^{i} \partial z_{h}^{j}}=\left(0 ; 0, \ldots, 0 ; 0, \ldots,(-1)^{\sigma_{1}}, \ldots, 0 ; 0, \ldots, 0\right),
\end{gathered}
$$

и, следовательно,

$$
\frac{\partial R}{\partial z_{t}^{i}}=\frac{\partial R_{1}}{\partial z_{t}^{i}}, \quad \frac{\partial^{2} R}{\partial z_{t}^{i} \partial z_{h}^{j}}=\frac{\partial^{2} R_{1}}{\partial z_{t}^{i} \partial z_{h}^{j}}-\delta^{i j} \delta_{t h} R_{1}
$$

Касательное пространство $T_{P} G^{+}(r, n+r)$ в $E^{N}$ состоит из векторов вида $(0 ; * ; 0, \ldots, 0 ; 0, \ldots, 0)$. Вектор $R_{1}$ является нормалью к гиперсфере $S^{N-1} \subset E^{N}$, в которую вложено $G^{+}(r, n+r)$. Поэтому легко видеть, что проекция $d^{2} R$ на нормальное пространство подмногообразия $G^{+}(r, n+r) \subset S^{N-1}$ в точке $P$ есть $\left(0 ; 0, \ldots, 0 ; \Omega_{4} ; 0, \ldots, 0\right)$, где $\Omega_{4}$ - набор координат $(-1)^{\sigma}\left(d z_{t}^{i} \cdot d z_{h}^{j}-d z_{h}^{i} \cdot d z_{t}^{j}\right), i, j=\overline{1, n}, t, h=\overline{1, r}$, и условие равенства нулю этой проекции эквивалентно выполнению условия (3.3), являющегося характеризационным свойством конуса касательных пространств стандартных сфер $S^{n}$ в $T_{P} G^{+}(r, n+r)$. Поэтому данньй конус является конусом асимптотических направлений в $T_{P} G^{+}(r, n+r)$ многообразия $G^{+}(r, n+r) \subset S^{N-1}$. Так как условие асимптотичности инвариантно при замене координат в $G^{+}(r, n+r)$, а точка $P$ выбиралась произвольно, то имеет место содержание утверждения леммы 1.

4. Если подмногообразие $F^{n}$ задано в декартовых координатах в $E^{n+r}$ гладкими $C^{p}, p>2$, функциями $x^{i}\left(v^{1}, \ldots, v^{n}\right)$, то легко видеть, что грассманов образ Г задается в $G^{+}(r, n+r)$ гладкими $C^{p-1}$ функциями $z_{j}^{i}\left(v^{1}, \ldots, v^{n}\right)$.

ЛЕмма 2. Если грассманов образ Г регулярного $C^{p}, p>2$, подмногообразия $F^{n}$ имеет размерность $k$, то на $F^{n}$ можсно ввести такие координаты $v^{i}, i=\overline{1, n}$, что $\partial z_{j}^{i} / \partial v^{t}=0, t=\overline{k+1, n}$.

ДокАЗАТЕЛьСтво. Пусть $F^{n}$ параметризовано координатами $u^{1}, \ldots$, $u^{n}$. Taк как $\operatorname{dim} \Gamma=k$, то можно считать, что

$$
\frac{\partial z}{\partial u^{t}}=\sum_{i=1}^{k} \alpha_{t}^{i} \frac{\partial z}{\partial u^{i}}, \quad t=\overline{k+1, n}
$$


и $\partial z / \partial u^{i}, i=\overline{1, k}$, линейно независимы. В координатах $v^{j}$ имеем

$$
\frac{\partial z}{\partial v^{j}}=\sum_{i=1}^{n} \frac{\partial z}{\partial u^{i}} \frac{\partial u^{i}}{\partial v^{j}}=\sum_{i=1}^{k}\left(\frac{\partial u^{i}}{\partial v^{j}}+\sum_{t=k+1}^{n} \alpha_{t}^{i} \frac{\partial u^{t}}{\partial v^{j}}\right) \frac{\partial z}{\partial u^{i}} .
$$

Найдем $u^{i}=u^{i}\left(v^{1}, \ldots, v^{n}\right)$ такие, что $\partial z / \partial v^{j}=0, j=\overline{k+1, n}$. Из (4.2) имеем

$$
\frac{\partial u^{i}}{\partial v^{j}}=-\sum_{t=k+1}^{n} \alpha_{t}^{i} \frac{\partial u^{t}}{\partial v^{j}}, \quad i=\overline{1, k}, \quad j=\overline{k+1, n} .
$$

Условие совместности системы (4.3) имеет вид

$$
\begin{aligned}
& \sum_{t=k+1}^{n} \sum_{h=1}^{k}\left(\frac{\partial \alpha_{t}^{i}}{\partial u^{h}} \frac{\partial u^{h}}{\partial v^{g}} \frac{\partial u^{t}}{\partial v^{j}}-\frac{\partial \alpha_{t}^{i}}{\partial u^{h}} \frac{\partial u^{h}}{\partial v^{j}} \frac{\partial u^{t}}{\partial v^{g}}\right) \\
& +\sum_{t, h=k+1}^{n}\left(\frac{\partial \alpha_{t}^{i}}{\partial u^{h}}-\frac{\partial \alpha_{h}^{i}}{\partial u^{t}}\right) \frac{\partial u^{h}}{\partial v^{g}} \frac{\partial u^{t}}{\partial v^{j}}=0, \quad i=\overline{1, k}, \quad j, g=\overline{k+1, n} .
\end{aligned}
$$

Подставляя в первую группу слагаемых вместо $\partial u^{h} / \partial v^{g}$ и $\partial u^{h} / \partial v^{j}$ их выражения из (4.3) и меняя порядок суммирования там, где это нужно, можно привести систему (4.4) к виду

$$
\sum_{t, h=k+1}^{n} \Phi_{h t}^{i} \frac{\partial u^{h}}{\partial v^{g}} \frac{\partial u^{t}}{\partial v^{j}}=0, \quad i=\overline{1, k}, \quad i, g=\overline{k+1, n}
$$

где

$$
\Phi_{h t}^{i}=\frac{\partial \alpha_{t}^{i}}{\partial u^{h}}-\frac{\partial \alpha_{h}^{i}}{\partial u^{t}}+\sum_{m=1}^{k}\left(\frac{\partial \alpha_{h}^{i}}{\partial u^{m}} \alpha_{t}^{m}-\frac{\partial \alpha_{t}^{i}}{\partial u^{m}} \alpha_{h}^{m}\right) .
$$

Так как по условию $z_{j}^{i}$ являются гладкими $C^{p}, p>1$, функциями, то $\partial^{2} z / \partial u^{i} \partial u^{j}=\partial^{2} z / \partial u^{j} \partial u^{i}$ и из (4.1) получаем

$$
\begin{gathered}
\sum_{i=1}^{k}\left(\frac{\partial \alpha_{g}^{i}}{\partial u^{j}} \frac{\partial z}{\partial u^{i}}+\alpha_{g}^{i} \frac{\partial^{2} z}{\partial u^{i} \partial u^{j}}-\frac{\partial \alpha_{j}^{i}}{\partial u^{g}} \frac{\partial z}{\partial u^{i}}-\alpha_{j}^{i} \frac{\partial^{2} z}{\partial u^{i} \partial u^{g}}\right)=0 \\
j, g=\overline{k+1, n} .
\end{gathered}
$$

Из (4.1) найдем выражение для $\partial^{2} z / \partial u^{i} \partial u^{j}$. Подставляя его в (4.6) и меняя порядок суммирования там, где это необходимо, получаем

$$
\begin{aligned}
\sum_{i, t=1}^{k}\left(\frac{\partial \alpha_{g}^{i}}{\partial u^{j}}-\frac{\partial \alpha_{j}^{i}}{\partial u^{g}}\right. & \left.+\alpha_{g}^{t} \frac{\partial \alpha_{j}^{i}}{\partial u^{t}}-\alpha_{j}^{t} \frac{\partial \alpha_{g}^{i}}{\partial u^{t}}\right) \\
& +\sum_{i, t=1}^{k}\left|\begin{array}{cc}
\alpha_{g}^{i} & \alpha_{j}^{i} \\
\alpha_{g}^{t} & \alpha_{j}^{t}
\end{array}\right| \frac{\partial^{2} z}{\partial u^{i} \partial u^{t}}=0, \quad j, g=\overline{k+1, n} .
\end{aligned}
$$


Замечая, что вторая группа слагаемых равна нулю в силу симметричности $\partial^{2} z / \partial u^{i} \partial u^{t}$ и кососимметричности $\alpha_{j}^{i} \alpha_{g}^{t}-\alpha_{g}^{i} \alpha_{j}^{t}$ по индексам $i$ и $t$, а также тот факт, что выражение в скобках в первой групше слагаемых есть $\Phi_{j g}^{i}$, получаем

$$
\sum_{i=1}^{k} \Phi_{j g}^{i} \frac{\partial z}{\partial u^{i}}=0, \quad j, g=\overline{k+1, n} .
$$

Но $\partial z / \partial u^{i}, i=\overline{1, k}$, линейно независимы, поэтому $\Phi_{j g}^{i}=0$, и, значит, система (4.5) вьполнена тождественно, а система (4.3) является совместной. Задавая $u^{k+1}, \ldots, u^{n}$ произвольно, а $u^{1}, \ldots, u^{k}$ вдоль подмногообразия $v^{i}=$ const, $i=\overline{1, k}$, на $F^{n}$, находим решение системы (4.3). Для регулярности параметризации $F^{n}$ координатами $v^{i}$ достаточно задать $u^{k+1}, \ldots$, $u^{n}$ и $u^{1}, \ldots, u^{k}$ вдоль $v^{i}=$ const, $i=\overline{1, k}$, так, чтобы вдоль $v^{i}=$ const, $i=\overline{1, k}$, вьполнялось условие $\operatorname{rg}\left\|\partial u^{i} / \partial v^{j}\right\|=n$. Тогда в некоторой окрестности подмногообразия $v^{i}=$ const, $i=\overline{1, k}$, параметризация $F^{n}$ координатами $v^{i}$ будет регулярна. Лемма 2 доказана.

Отметим, что в силу доказанной леммы подмногообразие $F^{n} \mathrm{c} k$-мерным грассмановым образом состоит из подмногообразий размерности $n-k$, вдоль которых нормальное пространство поверхности $F^{n} \subset E^{n+r}$ стационарно.

5. ДОКАЗАТЕЛЬСТВО ТЕОРЕМЫ. Переформулируем рассматриваемую проблему восстановления (см. п. 1) аналогично сделанному в [2]. Нам задана кривая $\Gamma \subset G^{+}(r, n+r) r$-мерных подпространств $E^{r}\left(v^{1}\right) \subset E^{n+r}$, $v^{1} \in(a, b) \subset \mathbb{R}^{1}$. Так как рассмотрение носит локальньй характер, то можно считать, что все $E^{r}\left(v^{1}\right)$ проектируются без вырождения на подпространство $E_{1}^{r}$ векторов $e_{1}, \ldots, e_{r}$ ортонормированного базиса в $E^{n+r}$. В каждом $E^{r}\left(v^{1}\right)$ существует базис векторов $\varepsilon_{\alpha}=\left(\varepsilon_{\alpha}^{1}, \ldots, \varepsilon_{\alpha}^{n+r}\right)$ специального вида (см. п. 2). Вследствие леммы 2 на искомом подмногообразии $F^{n} \subset E^{n+r}$ существует локальная система координат $v^{1}, \ldots, v^{n}$, где $v=\left(v^{1}, \ldots, v^{n}\right) \in D,(D-$ область в пространстве параметров, проектирующаяся на прямую параметра $v^{1}$ в интервал $\left.(a, b)\right)$. Требуется найти $n+r$ функций $x^{i}\left(v^{1}, \ldots, v^{n}\right)$, удовлетворяющих в $D$ условиям

$$
\begin{gathered}
\sum_{i=1}^{n+r} \varepsilon_{\alpha}^{i} \frac{\partial x^{i}}{\partial v^{j}}=0, \quad \alpha=\overline{1, r}, \quad j=\overline{1, n}, \\
\operatorname{rg}\left\|\frac{\partial x^{i}}{\partial v^{j}}\right\|=n .
\end{gathered}
$$

$\Phi$ ункции $x^{i}$ как декартовы координаты в $E^{n+r}$ образуют тогда радиус-вектор поверхности $F^{n}$, для которой Г является грассмановьм образом в си- 
лу (5.1) и которая регулярна в силу (5.2). Учитывая (3.4), из (5.1) получаем

$$
\frac{\partial x^{\alpha}}{\partial v^{i}}=-\frac{\partial x^{r+1}}{\partial v^{i}} z_{\alpha}^{1}-\cdots-\frac{\partial x^{r+n}}{\partial v^{i}} z_{\alpha}^{n}, \quad \alpha=\overline{1, r}, \quad i=\overline{1, n} .
$$

Условие (5.2) невырожденности касательного пространства поверхности $F^{n} \subset E^{n+r}$ очевидно эквивалентно (в силу локальности рассматриваемой задачи) условию невырожденности проекции касательного пространства на ортогональное дополнение подпространства $E_{1}^{r} \subset E^{n+r}$, имеющему вид:

$$
\operatorname{det} \dot{J} \neq 0,
$$

где $\dot{J}$ - матрица с элементами $\dot{J}_{j}^{i}=\partial x^{r+i} / \partial v^{j}, i, j=\overline{1, n}$. Так как заданные функции $z_{j}^{i}$ не зависят от $v^{2}, \ldots, v^{n}$, то условие совместности системы (5.3) имеет вид:

$$
\frac{\partial x^{r+1}}{\partial v^{i}} \frac{\partial z_{\alpha}^{1}}{\partial v^{1}}+\cdots+\frac{\partial x^{r+n}}{\partial v^{i}} \frac{\partial z_{\alpha}^{n}}{\partial v^{1}}=0, \quad \alpha=\overline{1, r}, \quad i=\overline{2, n} .
$$

Если обозначать через $z_{\alpha}$ вектор-столбцы матрицы $z$, а через $y$ - вектор с координатами $y^{i}=x^{r+i}, i=\overline{1, n}$, то (5.5) можно записать в виде

$$
\left(\frac{\partial z_{\alpha}}{\partial v^{1}}, \frac{\partial y}{\partial v^{i}}\right)=0, \quad \alpha=\overline{1, r}, \quad i=\overline{2, n}
$$

где $(*, *)$ - скалярное произведение в евклидовом пространстве.

Предположим, что в некоторой точке $v_{1} \in D$ существуют $\alpha, \beta$ такие, что простой бивектор $\left[\partial z_{\alpha} / \partial v^{1}, \partial z_{\beta} / \partial v^{1}\right] \neq 0$. Тогда $n-1 n$-мерных векторов $\partial y / \partial v^{i}, i=\overline{2, n}$, в силу (5.6) должны быть ортогональны двум неколинеарным векторам $\partial z_{\alpha} / \partial v^{1}, \partial z_{\beta} / \partial v^{1}$ и, значит, быть линейно зависимыми. Поэтому в точке $v_{1} \in D$ будет нарушено условие (5.4). Следовательно, если $\Gamma$ - грассманов образ регулярного $F^{n} \subset E^{n+r}$, то вдоль $\Gamma$ выполнено условие

$$
\forall \alpha, \beta \quad\left[\frac{\partial z_{\alpha}}{\partial v^{1}}, \frac{\partial z_{\beta}}{\partial v^{1}}\right]=0,
$$

и поэтому для каждой точки $Q \in \Gamma$ сушествует стандартная сфера $S^{n}$, содержащая $Q$, которой кривая $Г$ касается (см. п. 3). В силу леммы 1 линия $\Gamma$ будет асимптотической на многообразии $G^{+}(r, n+r) \subset S^{N-1}$; первая часть теоремы доказана.

Докажем вторую часть теоремы. Легко видеть, что из условия асимптотичности $\Gamma$ следует, что в каждой точке $Q \in \Gamma$ имеет место (5.7) и, значит, существует орт (в евклидовом смысле) $T\left(v^{1}\right)$ с координатами $T^{i}\left(v^{1}\right)$ и функции $\lambda_{\alpha}\left(v^{1}\right)$ такие, что

$$
\frac{\partial z_{\alpha}}{\partial v^{1}}=\lambda_{\alpha} T, \quad \alpha=\overline{1, r} .
$$


Система (5.5) примет тогда следуюший вид

$$
T^{1} \frac{\partial x^{r+1}}{\partial v^{i}}+\cdots+T^{n} \frac{\partial x^{r+n}}{\partial v^{i}}=0, \quad i=\overline{2, n} .
$$

В силу регулярности $\Gamma$ вектор $T \neq 0$. Возьмем произвольную точку $P$ с координатой $v_{0}^{1}$ на $\Gamma$; в области $D$ возьмем точку $v_{0}=\left(v_{0}^{1}, \ldots, v_{0}^{n}\right)$. Hе уменьшая общности, можно считать, что $T^{1}\left(v_{0}^{1}\right) \neq 0$. Тогда в некоторой окрестности $U\left(v_{0}\right) \subset D$ точки $v_{0}$ система (5.9) эквивалентна следующей:

$$
\frac{\partial x^{r+1}}{\partial v^{i}}=-\frac{T^{2}}{T^{1}} \cdot \frac{\partial x^{r+2}}{\partial v^{i}}-\cdots-\frac{T^{n}}{T^{1}} \cdot \frac{\partial x^{r+n}}{\partial v^{i}}, \quad i=\overline{2, n} .
$$

Так как $T$ не зависит от $v^{2}, \ldots, v^{n}$, то, очевидно, (5.10) совместна. Задавая $x^{r+2}, \ldots, x^{r+n}$ в $U\left(v_{0}\right)$ и значения на прямой $\left(v^{1}, v_{0}^{2}, \ldots, v_{0}^{n}\right)$ в $U\left(v_{0}\right)$ в виде гладких $C^{p+1}$ функций так, чтобы в точке $v_{0}$ было вьполнено условие (5.4) (а это можно сделать, так как

$$
\operatorname{det} \dot{J}=\left(\sum_{i=1}^{n} T^{i} \frac{\partial x^{r+i}}{\partial v^{1}}\right) \frac{\operatorname{det} \dot{J}^{\prime}}{T^{1}}
$$

где $\dot{J}^{\prime}$ - минор матрицы $\dot{J}$, соответствующий элементу $\left.\dot{J}_{1}^{1}\right)$, находим в некоторой окрестности $U_{1}\left(v_{0}\right) \subset U\left(v_{0}\right)$ гладкое решение системы (5.10), удовлетворяющее (5.4). Подставляя найденные функции в (5.3), получаем для каждой функции ' $x^{i}\left(v^{1}, \ldots, v^{n}\right), i=\overline{1, r}$, совместную систему. Задавая значения искомых функций в точке $v_{0}$, получаем гладкое $C^{p+1}$ решение системы (5.3) в некоторой окрестности $U_{2}\left(v_{0}\right) \subset U_{1}\left(v_{0}\right)$. Таким образом, найдена окрестность точки $P$ на $\Gamma$, отвечающая интервалу изменения параметра $v^{1}$, когда $v \in U_{2}\left(v_{0}\right)$, и регулярное подмногообразие $F^{n} \subset E^{n+r}$ с радиус-вектором $\mathbf{r}(v)=\left(x^{1}(v), \ldots, x^{n+r}(v)\right)$, для которого Г является грассмановым образом. Теорема доказана.

ДоКАЗАТЕЛЬСТВО СЛЕДСТВИЯ. Так как $T$ зависит только от $v^{1}$, то систему (5.9) можно проинтегрировать:

$$
x^{r+1} T^{1}+\cdots+x^{r+n} T^{n}=f\left(v^{1}\right),
$$

т.е. для любого решения системы (5.9) существует функция $f\left(v^{1}\right)$ вида (5.11), и для любой функции $f\left(v^{1}\right)$ функции $x^{r+i}, i=\overline{1, n}$, удовлетворяющие (5.11), являются решением системы (5.9). Уравнения (5.3) при $i=\overline{2, n}$ интегрируются аналогичным образом:

$$
x^{\alpha}=-x^{r+1} z_{\alpha}^{1}-\cdots-x^{r+n} z_{\alpha}^{n}+g^{\alpha}\left(v^{1}\right), \quad \alpha=\overline{1, r} .
$$


Подставляя (5.12) в уравнения (5.3) при $i=1$ и учитьвая (5.11), получаем

$$
\frac{\partial g^{\alpha}}{\partial v^{1}}=\lambda_{\alpha}\left(v^{1}\right) f\left(v^{1}\right), \quad \alpha=\overline{1, r} .
$$

При заданных функциях $x^{r+i}(v), i=\overline{1, n}$, для любого решения системы (5.3) существуют функции $g^{\alpha}\left(v^{1}\right)$ из (5.12), удовлетворяющие (5.13). И обратно, для любых функций $g^{\alpha}\left(v^{1}\right)$, удовлетворяющих (5.13), функции $x^{1}(v), i=\overline{1, r}$, из (5.12) являются решением системы (5.3). Тогда радиус-вектор искомого подмногообразия $F^{n} \subset E^{n+r}$ имеет вид

$$
\mathbf{r}\left(v^{1}, \ldots, v^{n}\right)=\sum_{i=1}^{n}\left(\begin{array}{c}
-z_{1}^{i} \\
\vdots \\
-z_{r}^{i} \\
\delta_{1}^{i} \\
\vdots \\
\delta_{n}^{i}
\end{array}\right) x^{r+i}+\left(\begin{array}{c}
g^{1} \\
\vdots \\
g^{r} \\
0 \\
\vdots \\
0
\end{array}\right)
$$

где $x^{r+i}$ удовлетворяют (5.11), а $g^{\alpha}-(5.13)$. Предположим, что как и ранее $T^{1} \neq 0$. Тогда легко получить, что $\operatorname{det} \dot{J}=\operatorname{det} \dot{J}^{\prime} f\left(v^{1}\right) / T^{1} \neq 0$ в силу регулярности $F^{n}$. Заменяя $v^{2}, \ldots, v^{n}$ на $x^{r+2}, \ldots, x^{r+n}$, и выражая $x^{r+1}$ из (5.11), получаем из (5.14):

$$
\mathbf{r}\left(v^{1}, x^{r+2}, \ldots, x^{r+n}\right)=\omega_{1}\left(v^{1}\right)+\sum_{i=2}^{n} \omega_{i}\left(v^{1}\right) x^{r+i},
$$

где векторы $\omega_{i}$ имеют следующую форму

$$
\omega_{1}=\left(\begin{array}{c}
g^{1}-z_{1}^{1} \frac{f}{T^{1}} \\
\vdots \\
g^{r}-z_{r}^{1} \frac{f}{T^{1}} \\
\frac{f}{T^{1}} \\
0 \\
\vdots \\
0
\end{array}\right), \quad \omega_{i}=\frac{1}{T^{1}}\left(\begin{array}{c}
z_{1}^{1} T^{i}-z_{1}^{i} T^{1} \\
\vdots \\
z_{r}^{1} T^{i}-z_{r}^{i} T^{1} \\
-T^{i} \\
\delta_{i}^{2} T^{1} \\
\vdots \\
\delta_{i}^{n} T^{1}
\end{array}\right), \quad i=\overline{2, n}
$$

т.е. $\omega_{i}, i=\overline{2, n}$, полностью определяются заданием кривой $\Gamma \subset G^{+}(r, n+r)$, а вектор $\omega_{1}$ определяется с некоторым произволом. Радиус-вектор (5.14) имеет наглядное геометрическое толкование. По заданному 1-параметрическому семейству $r$-мерных подпространств $E^{r}\left(v^{1}\right) \subset E^{n+r}$, проектирующихся без вырождения на подпространство $E_{1}^{r}$, строится семейство 
$n$-мерных ортогональньх дополнений $E^{n}\left(v^{1}\right)$. Условием (5.11) в каждом $E^{n}\left(v^{1}\right)$ выделяется гиперплоскость $E^{n-1}\left(v^{1}\right)$. В $E_{1}^{r}$ строится кривая с радиус-вектором $\rho=\left(g^{1}, \ldots, g^{r}, 0, \ldots, 0\right)$ и каждое $E^{n}\left(v^{1}\right)$ переносится в соответствующую точку $\rho\left(v^{1}\right)$ кривой параллельно. Искомоеподмногообразие $F^{n} \subset E^{n+r}$ образовано соответствуюшими плоскостями $E^{n-1}\left(v^{1}\right)$ и вдоль каждой образующей нормальное пространство подмногообразия $F^{n} \subset E^{n+r}$ стационарно.

Физико-технический институт Поступило низких температур АН Украины 27.09 .93

E-mail : gorkaviy@ilt.kharkov.ua

\section{СПИСОК ЦИТИРОВАННОЙ ЛИТЕРАТУРЫ}

[1] Аминов Ю. А. Определение поверхности в четырехмерном евклидовом пространстве по ее грассманову образу // Матем. сб. 1982. Т. 117. № 2. С. 147-160.

[2] Аминов Ю. А. О грассмановом образе двумерной поверхности в четырехмерном евклидовом пространстве // Укр. геом. сб. 1980. № 23. С. 3-16.

[3] Hoffman D., Osserman R. The Gauss map of surfaces in $\mathbb{R}^{3}$ and $\mathbb{R}^{4} / /$ Proc. London Math. Soc. 1985. V. 50. № 1. P. 27-56.

[4] Борисенко А. А. О полных параболических поверхностях // Укр. геом. сб. 1985. № 28. C. 8-19.

[5] Аминов Ю. А., Тарасова Т. С. Определение поверхности в $E$ по вырожденному грассманову образу // Укр. геом. сб. 1983. № 26. С. 6-13.

[6] Рохлин В. А., Фукс Д. Б. Начальньй курс топологии. Геометрические главы. М.: Наука, 1977.

[7] Борисенко А.А., Николаевский Ю.А. Многообразия Грассмана и грассманов образ подмногообразий // УМН. 1991. Т. 46. № 2(278). С. 41-83.

[8] Николаевский Ю.А. Вполне омбилические подмногообразия в $G(2, n)$. I // Укр. геом. сб. 1991. №34. С. 83-98. 